\title{
$\Delta|\mathrm{P}|$ chaos \\ An Interdisciplinary Journal of Nonlinear Science
}

\section{Transition to chemical turbulence}

Q. Ouyang and Harry L. Swinney

Citation: Chaos 1, 411 (1991); doi: 10.1063/1.165851

View online: http://dx.doi.org/10.1063/1.165851

View Table of Contents: http://scitation.aip.org/content/aip/journal/chaos/1/4?ver=pdfcov

Published by the AIP Publishing

\section{Articles you may be interested in}

Fractal lifetimes in the transition to turbulence

Chaos 14, S11 (2004); 10.1063/1.1821751

Stochastic Transition of a Turbulent Plasma

AIP Conf. Proc. 669, 228 (2003); 10.1063/1.1593907

The transition to turbulence

Phys. Today 31, 41 (1978); 10.1063/1.2995142

Transition and Turbulence in Hypersonic Wakes

Phys. Fluids 9, 446 (1966); 10.1063/1.1761696

On the Mechanism of Turbulent Transition

Phys. Fluids 3, 1026 (1960); 10.1063/1.1706139

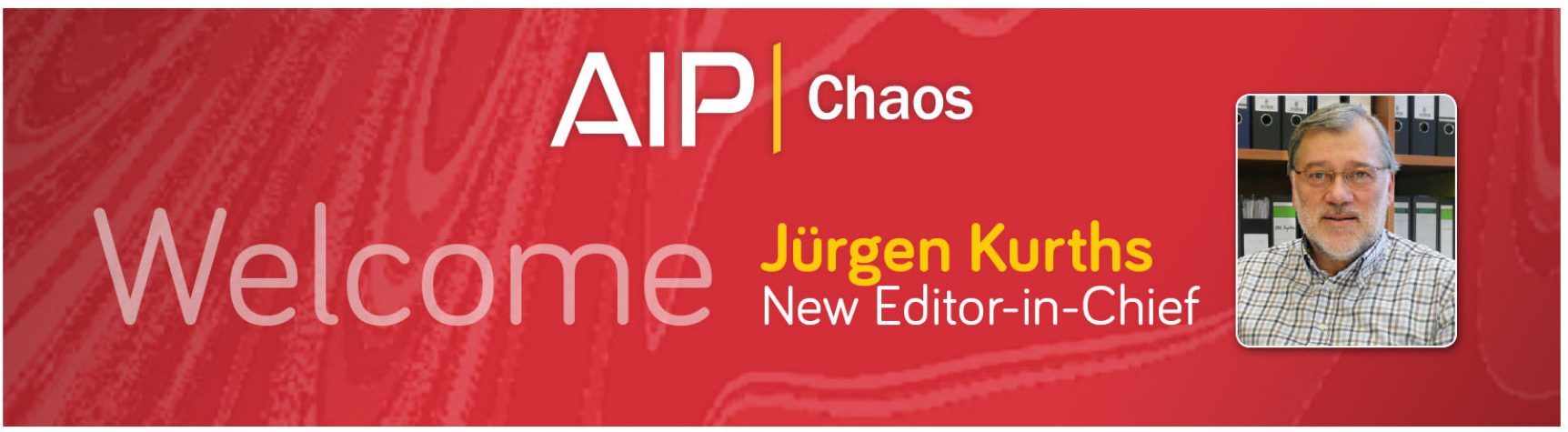




\title{
Transition to chemical turbulence
}

\author{
Q. Ouyang and Harry L. Swinney \\ Center for Nonlinear Dynamics and Department of Physics, University of Texas, Austin, Texas 78712
}

(Received 12 August 1991; accepted for publication 30 September 1991)

Experiments have been conducted on Turing-type chemical spatial patterns and their variants in a quasi-two-dimensional open spatial reactor with a chlorite-iodide-malonic acid reaction. A variety of stationary spatial structures-hexagons, stripes, and mixed states-were observed, and transitions to these states were studied. For conditions beyond those corresponding to the emergence of patterns, a transition was observed from stationary spatial patterns to chemical turbulence, which is marked by a continuous motion of the pattern within a domain and of the grain boundaries between domains. The transition to chemical turbulence was analyzed by measuring the correlation length, the average pattern speed, and the total length of the domain boundaries. The emergence of chemical turbulence is accompanied by a large increase in the defects in the pattern, which suggests that this is an example of defect-mediated turbulence.

\section{INTRODUCTION}

Topological or defect-mediated turbulence arises when a regular stationary or spatiotemporal pattern loses its stability as a control parameter is varied. This phenomenon has been observed in a number of systems, including Rayleigh-Bénard convection, ${ }^{1,2}$ electrohydrodynamic convection in nematic liquid crystals, ${ }^{3}$ and surface waves. ${ }^{4,5}$ Recent theoretical studies indicate that the phenomenon is ubiquitous and calls for an essentially system-free interpretation. ${ }^{6,7}$ However, in chemical reaction-diffusion systems, despite extensive theoretical and numerical studies of patterns in the past two decades, ${ }^{8-15}$ there has been no report on the observation of chemical turbulence in an open system, either in laboratory experiments or numerical simulations. ${ }^{16}$

The spontaneous formation of stationary spatial chemical patterns from an initially uniform state was predicted in 1952 by Turing, ${ }^{17}$ but not observed in controlled laboratory experiments until 1990, when Castets et al. ${ }^{18-20}$ discovered stationary patterns in a concentration gradient in a gel reactor. Those experiments, which were conducted for a chlorite-iodide-malonic acid reaction, showed hexagonal and striped patterns localized in a band. Defects in the hexagonal patterns were also observed and studied, ${ }^{19}$ but no chemical turbulence was reported.

Here, we report observations of extended two-dimensional Turing patterns (hexagons, stripes, and a mixed state) in a disc gel reactor that is initially uniform in the plane of the disk and has a gradient in the third dimension (see also our brief report in Ref. 21). Figure 1(a) shows an example of such spatial structures-a hexagonal pattern. These patterns are formed, as a control parameter is varied, in a well-defined transition from a spatially uniform state. For conditions well beyond those corresponding to the emergence of Turing patterns, another bifurcation is observed to lead to spatiotemporal turbulence; an example of such a state is shown in Fig. 1(b). This chemical turbulence is fundamentally different from the spatiotemporal chaos observed for front structures in the Couette reactor. ${ }^{22-24}$ In the case of the front structures, the system is temporally chaotic but spatially highly coherent, while in our system the observed time-dependent patterns have no long-range spatial order. These patterns provide the first evidence of defect-mediated turbulence in a reactiondiffusion system.

Section II describes the experimental system. Section III presents results of experiments on the formation of stationary Turing patterns. Section IV describes the observations and characterization of chemical turbulence. Section $\mathrm{V}$ discusses the results.

\section{EXPERIMENTAL SYSTEM}

Figure 2 is a schematic diagram of our open spatial reactor, which is similar to the one used by Kshirsagar et $a l^{25}$ The core of the reactor is a polyacrylamide gel disk, $2.0 \mathrm{~mm}$ thick and $25.4 \mathrm{~mm}$ in diameter. The gel, which was prepared by the procedure described in Ref. 18, has about $95 \%$ void space and $80 \AA$ average pore size. This gel is sandwiched between two $0.4 \mathrm{~mm}$ thick, $25.4 \mathrm{~mm}$ diam thin porous glass disks (Vycor glass from Corning); see Fig. 2(a). The porous glass has about $25 \%$ void space and 100 $\AA$ average pore size. The gel and glass disks are transparent and chemically inert to the reaction. They prevent parasitic convective motion in the reaction medium but allow all reactants to diffuse freely in the system, except for the indicator, a soluble starch from Prolabo (Thiodène), which is immobile in the gel; the starch was preloaded into the gel during preparation. For our experimental conditions the gel was very stable. No chemical or physical changes were found in the gel after continuous experiments for 6 weeks, but the indicator faded a little during this long period. In the present experiments we replaced the gel every 4 weeks.

The outer flat surface of each porous glass disk is in contact with a compartment ( $1.8 \mathrm{ml}$ in volume) fed with chemicals from an external continuously fed stirred tank reactor (CSTR), $4.0 \mathrm{ml}$ in volume. The external CSTRs are fed from reservoirs A and B, respectively; see Fig. 
(a)

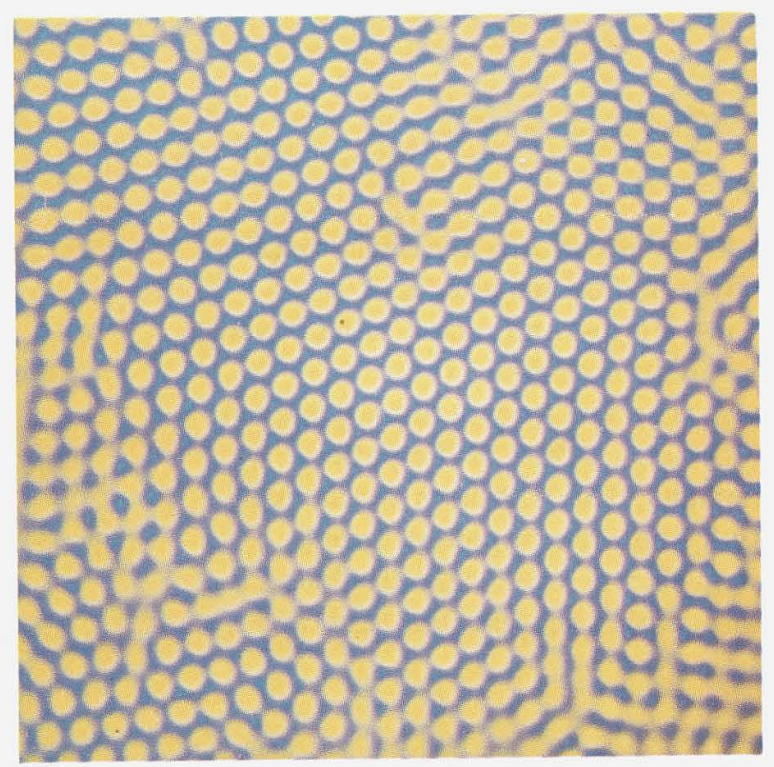

(b)

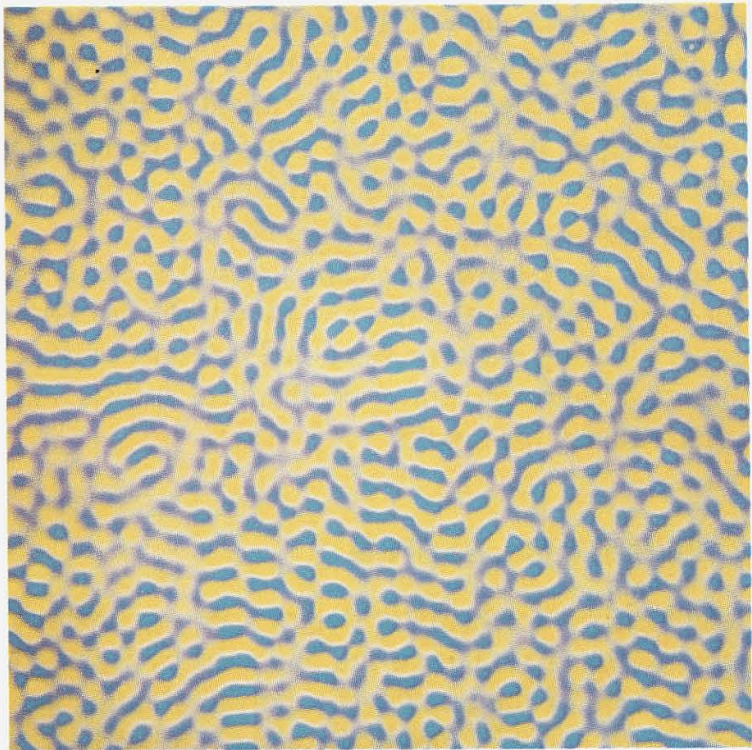

FIG. 1. (a) A stationary (Turing) chemical pattern, a hexagonal state; (b) a state of chemical turbulence. The concentration of sulfuric acid in reactor compartment B (see Fig. 2) was: in (a) $100 \mathrm{mM}$ and in (b) 8.5 $\mathrm{mM}$. Other control parameters were held fixed: $\left[\mathrm{I}^{-}\right]_{0}=3.0 \mathrm{mM}$, $\left[\mathrm{Na}_{2} \mathrm{SO}_{4}\right]_{0}=4.5 \mathrm{mM},\left[\mathrm{CH}_{2}(\mathrm{COOH})_{2}\right]_{0}^{\mathrm{B}}=9.0 \mathrm{mM},\left[\mathrm{ClO}_{2}{ }^{-}\right]_{0}^{\mathrm{A}}=18 \mathrm{mM}$, $\left[\mathrm{H}_{2} \mathrm{SO}_{4}\right]_{0}^{\mathrm{A}}=0.5 \mathrm{mM}$, temperature $=6.0^{\circ} \mathrm{C}$. The blue and yellow colors of the image correspond, respectively, to the reduced and oxidized states of the system. The region shown, about $8 \%$ of the total area, is $5.8 \times 5.8$ $\mathrm{mm}^{2}$

2(b). The residence time of the reagents in the compartments in contact with the porous glass must be much shorter than that of the reaction medium to insure uniform feed. For our system, the residence time of the former is 6.0 $\mathrm{s}$ and that of the latter is about $20 \mathrm{~min}$. The residence time of each CSTR was kept at $12 \mathrm{~min}$. The observed phenomena were found to be insensitive to the residence time of the CSTRs. Both the disk reactor and the CSTRs were immersed in a temperature-controlled water bath.

Different Vycor porous glass disks differed a few percent in thickness and diffusion coefficient values. Since the (a)

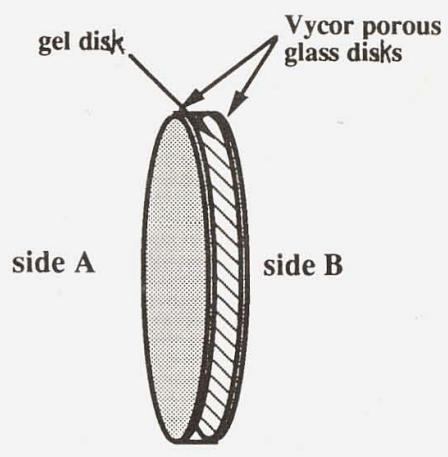

(b)

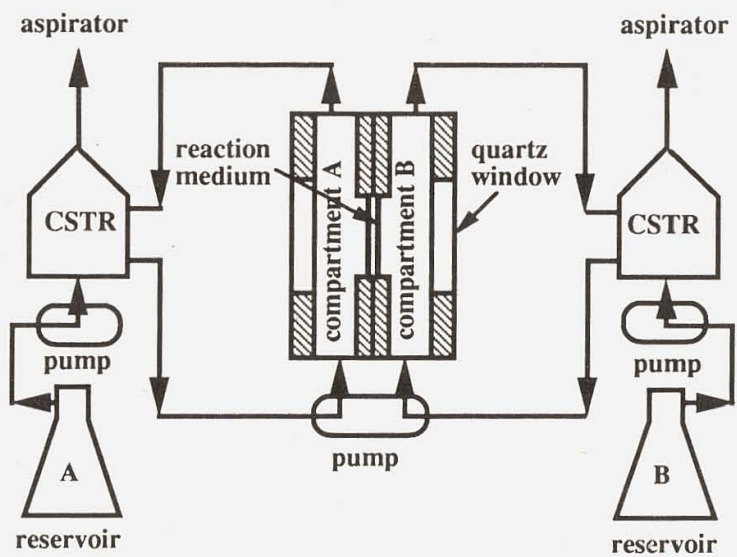

FIG. 2. Schematic diagram of an open spatial reactor: (a) reaction medium and (b) reaction system.

concentration gradient imposed across the $2.0 \mathrm{~mm}$ thickness of the gel is a function of the drop in concentration across the porous glass disks, a different thickness and diffusion coefficient of the porous glass results in a different concentration gradient imposed across the thickness of the gel disk. (The diffusion coefficients in the porous glass are about five times smaller than in the gel.) Hence, changing the porous glass disks shifts slightly the parameter regions where different types of patterns exist. In the extreme case we observed as much as a $25 \%$ shift in a chemical concentration corresponding to a transition in type of pattern when the porous glass disks were changed. Obviously, each bifurcation diagram was built using the same pair of glass disks.

The chemical system selected for our study is a chlorite-iodide-malonic acid reaction in an acidic (sulfuric acid) aqueous solution. This reaction is known to exhibit a rich nonlinear dynamic behavior: oscillations in a batch reactor or in a CSTR, ${ }^{26,27}$ transient target waves in a closed Petri dish, ${ }^{26}$ bistability in a CSTR, ${ }^{26-28}$ front structures in a Couette reactor, ${ }^{27,29-31}$ and Turing patterns in an open linear gel reactor. ${ }^{18-20}$ In the present experiments, components of the reaction were always distributed in the two compartments in such a way that neither compartment was separately reactive. Chlorite was only in "compartment A" 
and malonic acid was only in "compartment B"; thus there were opposing chemical concentration gradients in the direction normal to the plane of the gel. The other chemical species were contained in equal amounts in both reservoirs, except for sulfuric acid, which was more concentrated in compartment $B$ than in compartment A. Notice that chlorite and iodide in compartment $\mathrm{A}$ are at a low acid concentration; they would react rapidly at high acid concentration.

The chemicals diffuse through the porous glass disks into the gel where the reaction occurs. The gel, loaded with the starch indicator, changes in color from yellow to blue with changes in concentration of $\mathrm{I}_{3}^{-}$during the redox reaction. No starch is in the porous glass disks; thus concentration changes in the glass disks are not visible. The system was studied by changing stepwise (increasing or decreasing) one of the control parameters while fixing the others. The patterns were monitored in transmitted light ( $580 \mathrm{~nm}$, which is the wavelength of highest absorption of $\mathbf{I}_{3}^{-}$-starch complex). Enough time was allowed between changes of control parameter so that the system could relax to a new asymptotic state. This waiting time varied from 8 to $50 \mathrm{~h}$. Digitized black and white images were processed and analyzed on a Silicon Graphics work station.

Below the critical values of the control parameters, the system develops a chemical front in the direction of the concentration gradient imposed by the asymmetric boundary conditions at the surfaces of the gel. This chemical front separates the system into an oxidized region (side A, with low $\mathrm{I}_{3}^{-}$concentration and yellow color in the gel) and a reduced region (side $\mathrm{B}$, with high $\mathrm{I}_{3}^{-}$concentration and blue color in the gel) ${ }^{18}$ However, the system is uniform in the plane of the reaction medium perpendicular to the concentration gradient. The position of the chemical front in the direction normal to the plane of the gel depends on the control parameters; it is particularly sensitive to the concentration of chlorite in compartment $\mathrm{A}$, $\left[\mathrm{ClO}_{2}^{-}\right]_{0}^{\mathrm{A}}$. Here []$_{0}$ refers to the concentration of the mixed reactant stream in the compartment before any reaction takes place. The isont moves toward side $\mathrm{A}$ as $\left[\mathrm{ClO}_{2}^{-}\right]_{0}^{\mathrm{A}}$ decreases, and toward side $\mathrm{B}$ as $\left[\mathrm{ClO}_{2}^{-}\right]_{0}^{\mathrm{A}}$ increases. Since a reduced state in the gel gives a blue background that makes it impossible to observe spatial blue-yellow color patterns in transmitted light at $580 \mathrm{~nm}$, in most of our experiments we used $\left[\mathrm{ClO}_{2}^{-}\right]_{0}^{\mathrm{A}}$ as a tuning parameter in order to keep the chemical front near the boundary between the glass and the gel (near side B). Thus most of the reduced state is kept in the porous glass disk on side $\mathbf{B}$, which is transparent.

\section{TRANSITION TO TURING PATTERNS}

Beyond critical values of the control parameters, spatial patterns emerge spontaneously from the uniform chemical front structure. Initially, after the control parameters are switched into a regime where patterns arise, hundreds of transient yellow (light) circles grow in a blue (dark) background. Within an hour, these circles slowly stop propagating and self-organize into a transient honey- comb structure, as shown in Fig. 3(a). The yellow circles of the honeycomb structures gradually break up into short yellow stripes and then to a pattern of yellow dots that evolves more slowly. After $20 \mathrm{~h}$, the system settles down to a nearly stationary state of hexagonal patterns [see Fig. 3(b)] with many defects [see Fig. 3(e)]. Grain boundaries continue to move, but very slowly, typically only 0.2 $\mathrm{mm} /$ day.

For other control parameter values, e.g., a higher concentration of iodide, steady-state striped patterns were observed. In this case the short yellow stripes evolved from the honeycomb structure that gradually linked together, forming yellow stripes. The striped patterns, illustrated in Fig. 3(c) and (f), were stationary, in contrast to the widely studied traveling wave patterns (see, for example, Refs. 32 and 33). We monitored a striped pattern for days without observing any movement, except for a very slow motion of the grain boundaries that separate different domains of stripes; see Fig. 3(f).

In addition to the pure hexagons and stripes, a distinct stationary mixed state was also observed. Mixed states appeared as if they were an overlap of hexagons and stripes; see Fig. 3(d). The mixed states were quantitatively identified in a two-dimensional spatial Fourier transform, as illustrated in Fig. 4. Pure hexagons have six peaks of equal wave number and intensity, separated by angles of $60^{\circ} \pm 1^{\circ}$ with respect to the origin; see Fig. 4(a) ${ }^{34}$ Pure stripes have two peaks with equal wave number and equal intensity, separated by angles of $180^{\circ} \pm 1^{\circ}$; see Fig. 4(b). The mixed state has six equal-wave number peaks around the origin, separated by angles of $60^{\circ} \pm 1^{\circ}$, with one high-intensity peak followed by two low-intensity ones, then again one high and two low; see Fig. 4(c). In our experiments, the mixed states coexisted with hexagons or stripes (e.g., one can find a mixed state in Fig. 2(f)); we have not found a sharp transition point between this mixed state and other patterned states.

A transition from the uniform state to a hexagonal pattern was studied using temperature as a control parameter. The transition was found to be continuous and nonhysteretic (within the mesh in control parameter that was used, $0.5^{\circ} \mathrm{C}$ ), as discussed elsewhere. ${ }^{21}$ Using iodide concentration as the control parameter, we have now examined a transition from a striped state to a hexagonal state. As the concentration of iodide decreased (increased) past a critical value, which depended on other control parameters, hexagons (stripes) appeared at first at the grain boundaries, and then gradually spread out to cover the whole system. Near the transition, the pattern required about $30 \mathrm{~h}$ to reach an asymptotic state. No hysteresis was observed with steps of $1.0 \mathrm{mM}$ in iodide concentration.

The wavelength of the hexagonal and striped patterns was determined from spatial fast Fourier transforms and was found to vary continuously in the range $0.13-0.33 \mathrm{~mm}$ as control parameters were varied. Immediately after a change in a control parameter, the entire pattern would fade away and then a new pattern would emerge with a different wavelength. Thus the wavelength of the patterned state is an intrinsic property of the reaction-diffusion sys- 
(a)
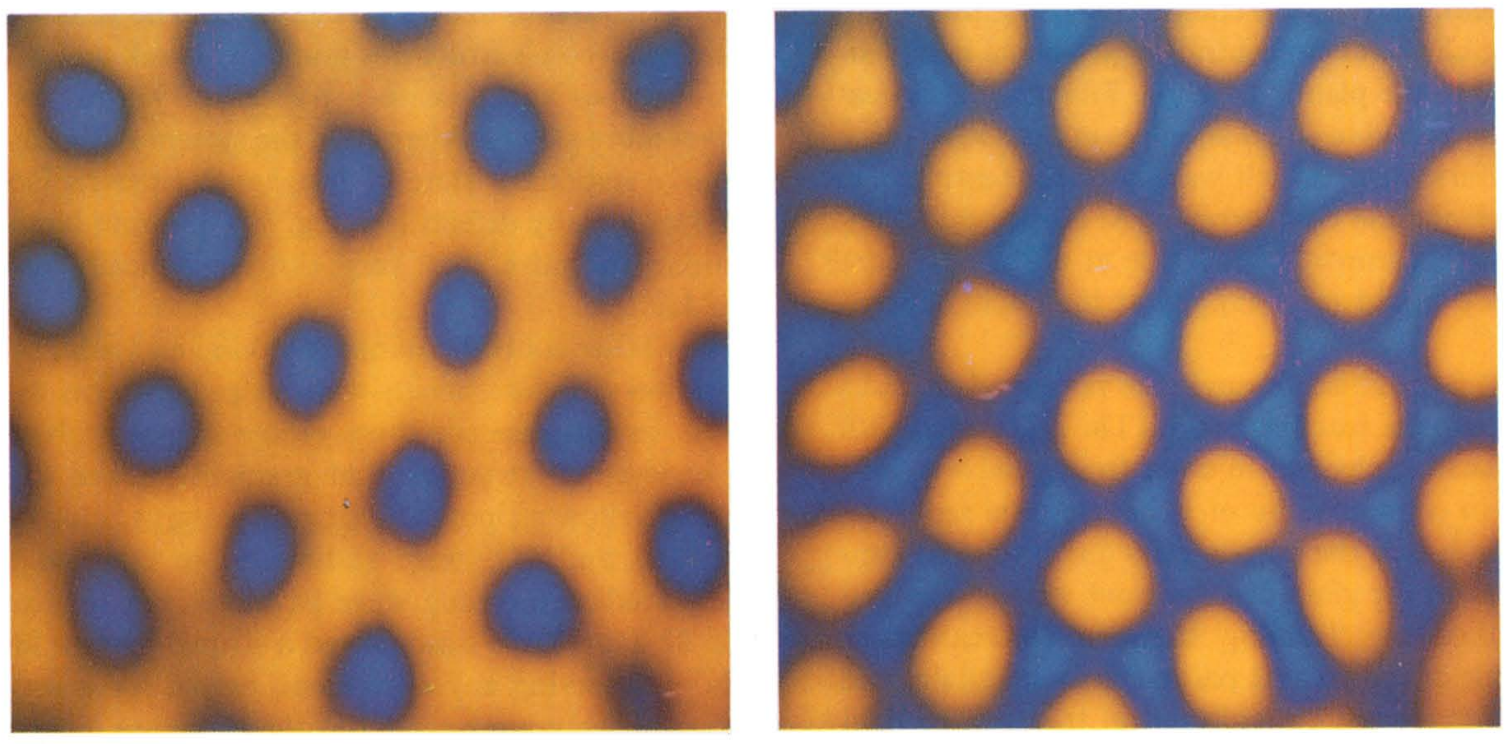

(b)

(c)
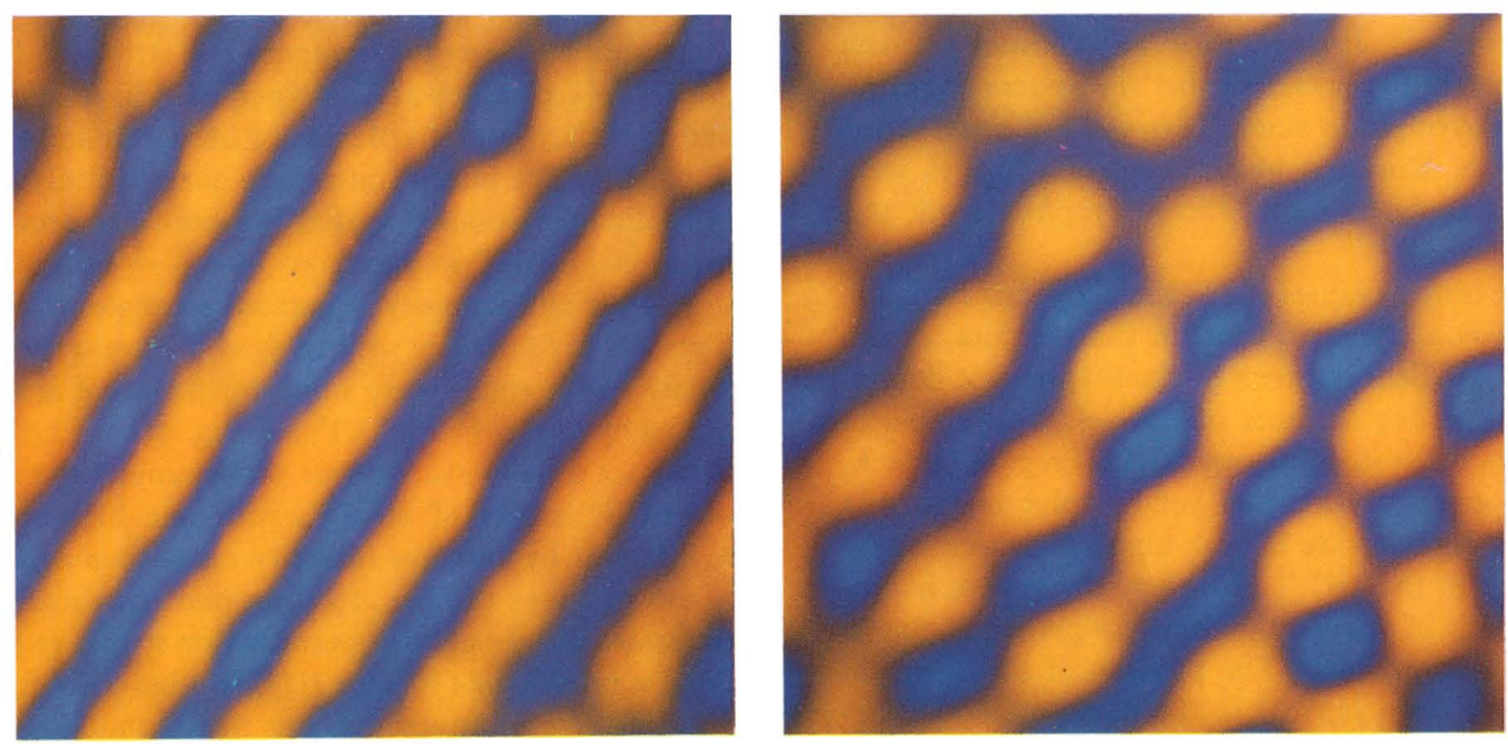

(d)

(e)
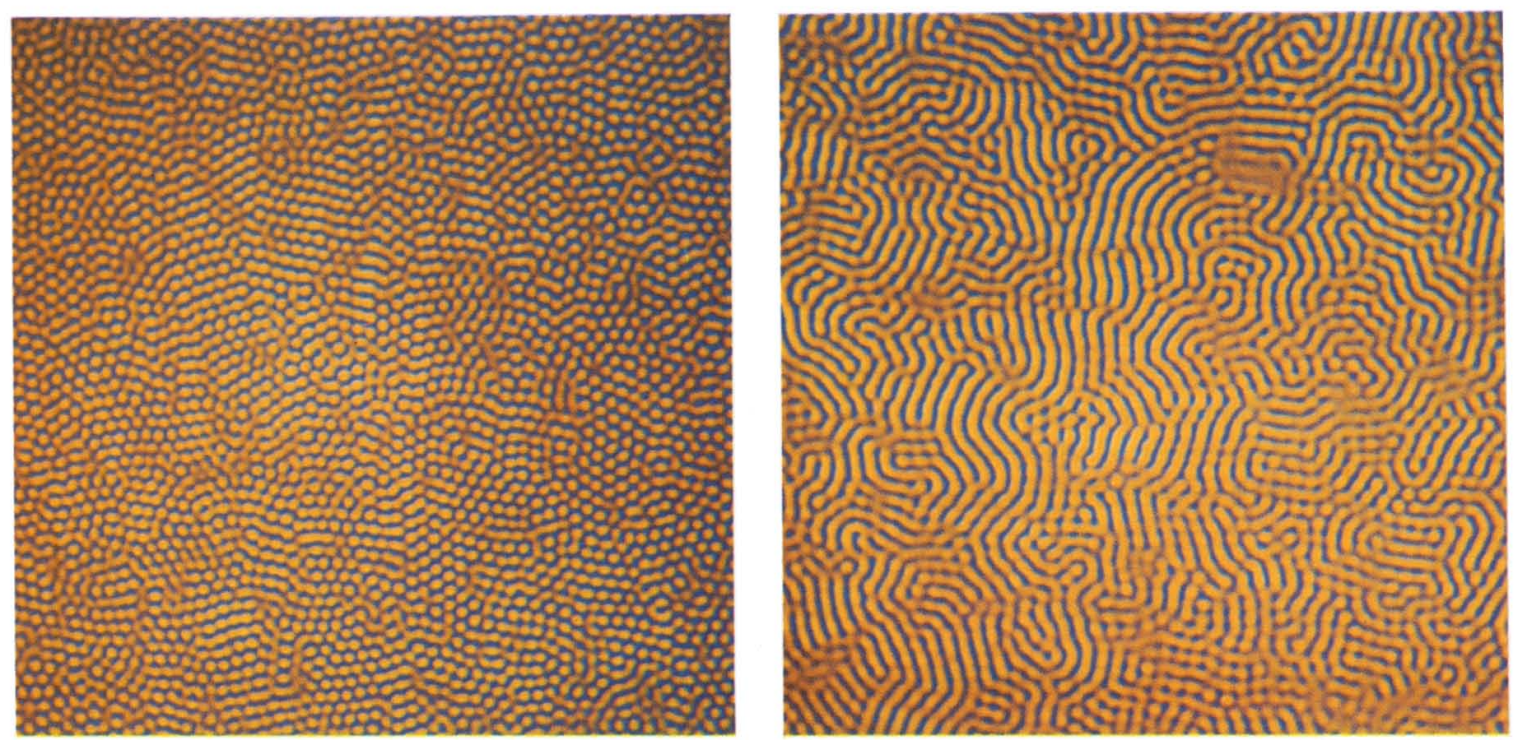

(f)

FIG. 3. Chemical patterns obtained with a continuously fed open spatial reactor: (a) transient honeycomb; (b) and (e), hexagons; (c) and (f), stripes; (d) mixed state. The bar beside each picture represents $1.0 \mathrm{~mm}$. The blue and yellow colors of the image correspond, respectively, to the reduced and oxidized states of the system. The concentrations in compartments $\mathrm{A}$ and $\mathrm{B}$ were: $\left[\mathrm{I}^{-}\right]_{0},\left[\mathrm{CH}_{2}(\mathrm{COOH})_{2}\right]_{0}^{\mathrm{B}},\left[\mathrm{ClO}_{2}^{-}\right]_{0}^{\mathrm{A}}$ (in $\left.\mathrm{mM}\right)$ in (a), (b), and (d), 3.5, $8.3,18.0$; in (c), 5.0, 8.3, 18.0; in (e), 3.0, 9.0, 12.0; in (f), 3.0, 11.0, 18.0. The control parameters common for all these patterns were: $\left[\mathrm{Na}_{2} \mathrm{SO}_{4}\right]_{0}=4.5$ $\mathrm{mM},\left[\mathrm{H}_{2} \mathrm{SO}_{4}\right]_{0}^{\mathrm{A}}=0.5 \mathrm{mM},\left[\mathrm{H}_{2} \mathrm{SO}_{4}\right]_{0}^{\mathrm{B}}=8.5 \mathrm{mM}$, temperature $=5.6^{\circ} \mathrm{C}$. 
(a)

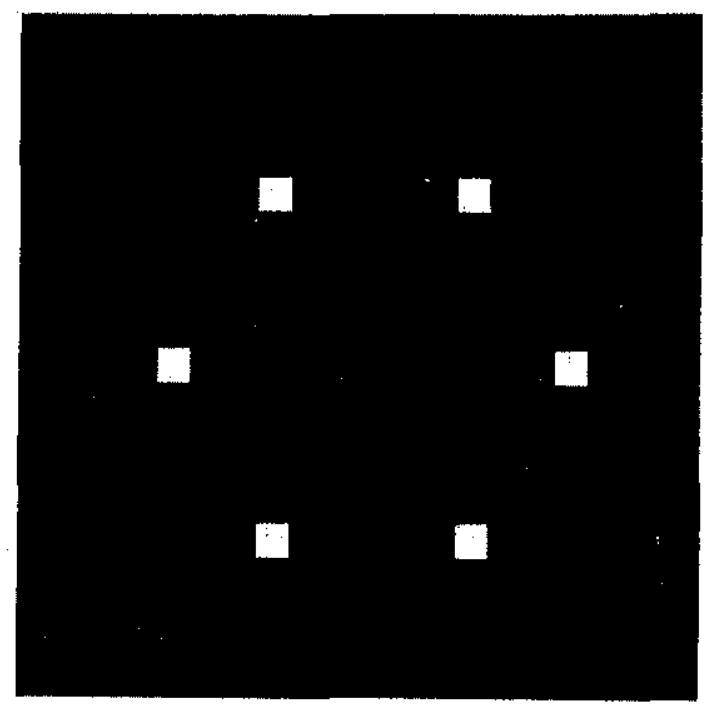

(b)

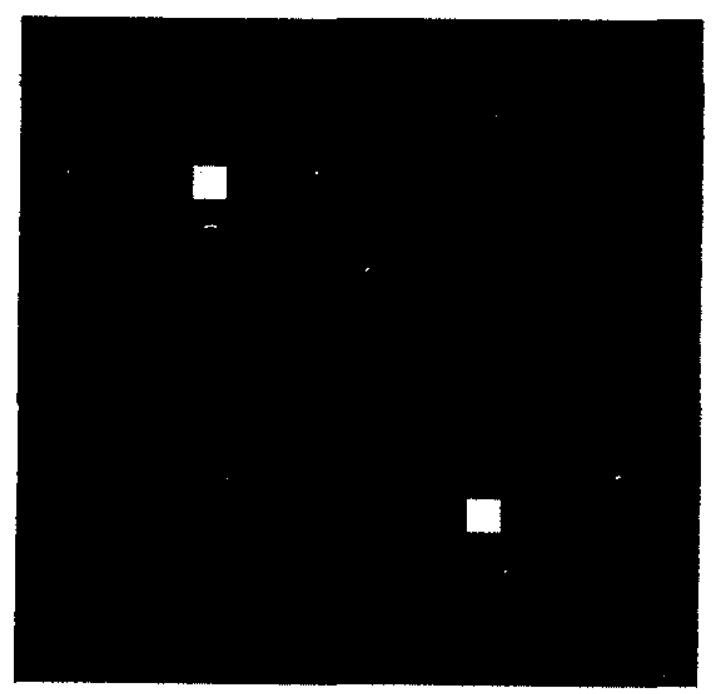

(c)

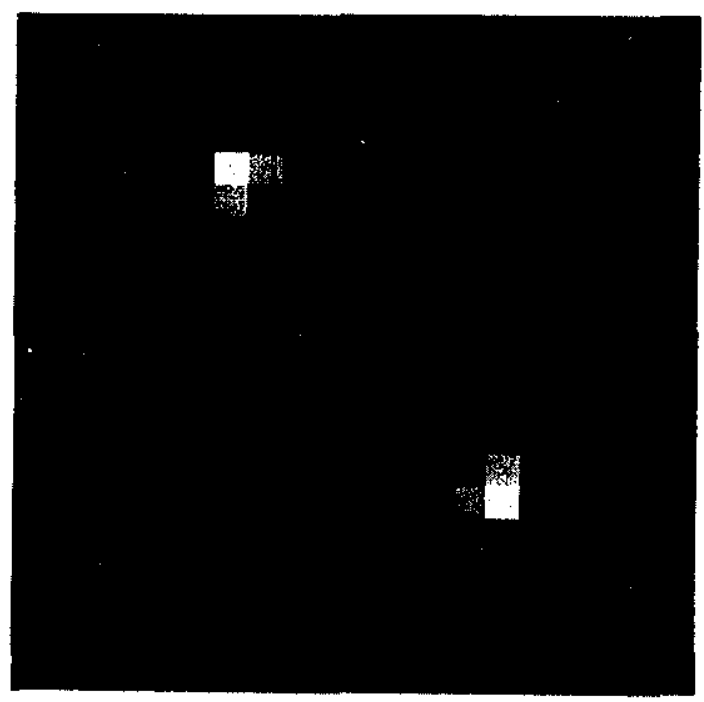

FIG. 4. Intensity power distributions of patterned states in spatial frequency space for (a) hexagons; (b) stripes; and (c) a mixed state. The grey level of the images increases from low to high as the intensity value (arbitrary units) changes from 0 to 255 . Conditions for (a), (b), and (c), respectively, correspond to the conditions in Fig. 3(b), (c), and (d).

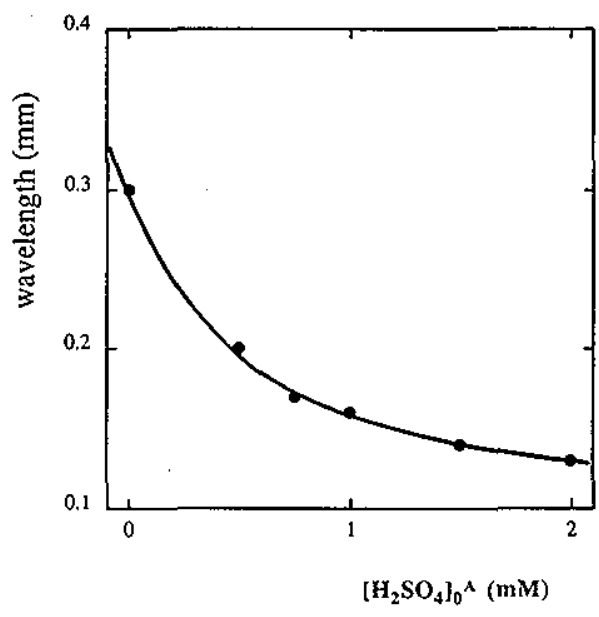

FIG. 5. Wavelength of a hexagonal pattern as a function of the sulfuric acid concentration in compartment $A$. The other control parameters were held fixed: $\left[\mathrm{I}^{-}\right]_{0}=3.5 \mathrm{mM},\left[\mathrm{Na}_{2} \mathrm{SO}_{4}\right]_{0}=4.5 \mathrm{mM},\left[\mathrm{CH}_{2}(\mathrm{COOH})_{2}\right]_{0}^{\mathrm{B}}$ $=8.3 \mathrm{mM},\left[\mathrm{ClO}_{2}^{-}\right]_{0}^{\mathrm{A}}=18 \mathrm{mM},\left[\mathrm{H}_{2} \mathrm{SO}_{4}\right]_{0}^{\mathrm{B}}=8.5 \mathrm{mM}$, temperature $=6.0^{\circ} \mathrm{C}$.

tem, not a consequence of the finite size of the system. This intrinsic wavelength distinguishes Turing patterns from other well-known nonequilibrium structures such as convection rolls ${ }^{35}$ or Taylor vortices. ${ }^{36}$ The wavelength of hexagons or stripes was found to be especially sensitive to the sulfuric acid concentration in compartment $\mathrm{A}$. For example, a decrease of $\left[\mathrm{H}_{2} \mathrm{SO}_{4}\right]_{0}^{\mathrm{A}}$ from $0.5 \mathrm{mM}$ to 0 led to a $45 \%$ increase in wavelength of the hexagons, as Fig. 5 illustrates. The position of spatial patterns in the third dimension also changes as a function of $\left[\mathrm{H}_{2} \mathrm{SO}_{4}\right]_{0}^{\mathrm{A}}$. For the experimental conditions of Fig. 5 , an increase of $\left[\mathrm{H}_{2} \mathrm{SO}_{4}\right]_{0}^{\mathrm{A}}$ above $2.0 \mathrm{mM}$ moved the position of spatial patterns in the third dimension from the gel into a porous glass disk, where spatial patterns were not visible.

The wavelength of the patterns was also found to be sensitive to malonic acid concentration. A decrease of malonic acid concentration for the striped state led to a decrease of the wavelength. However, when the malonic acid concentration was decreased past a critical value, which depended on other parameters, the striped state lost its stability and underwent a transition to another statechemical turbulence.

\section{TRANSITION TO CHEMICAL TURBULENCE}

Figure 1(b) shows a snapshot of a state of chemical turbulence. In contrast to the stationary patterns, such as hexagons or stripes, which have well-defined grain boundaries and a relatively small number of defects [see Fig. 3(e) and (f)], the state of chemical turbulence has a much larger number of defects that are distributed, apparently stochastically, throughout the system. These defects divide the total system into hundreds of small domains of dots and stripes; the length scale of each domain is only 2 to 3 wavelengths, much shorter than that of the domains found in the stationary hexagonal and striped states (see Fig. 3). Moreover, the turbulent patterns continuously evolve on a 


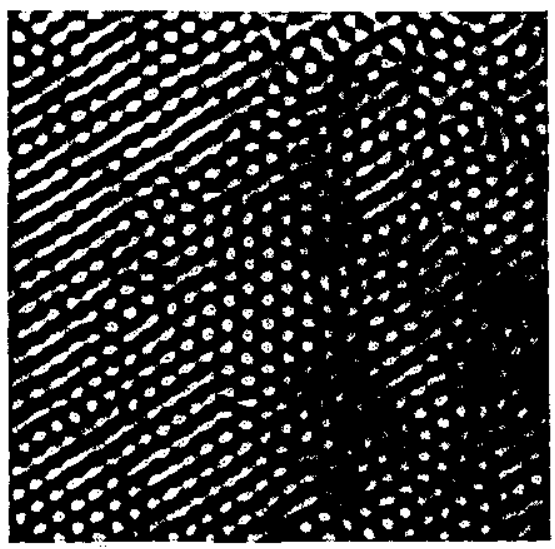

(a)

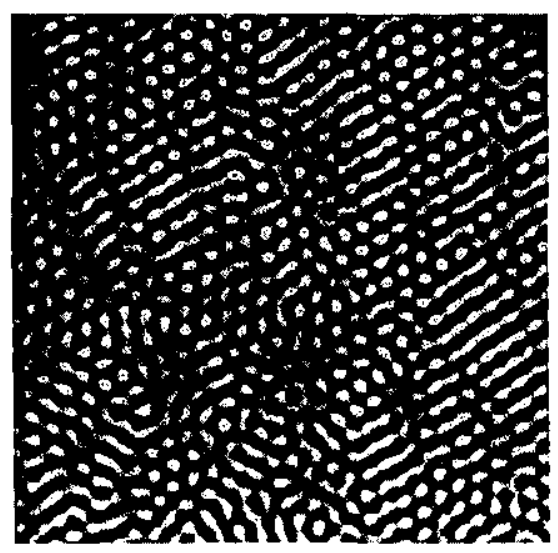

(b)

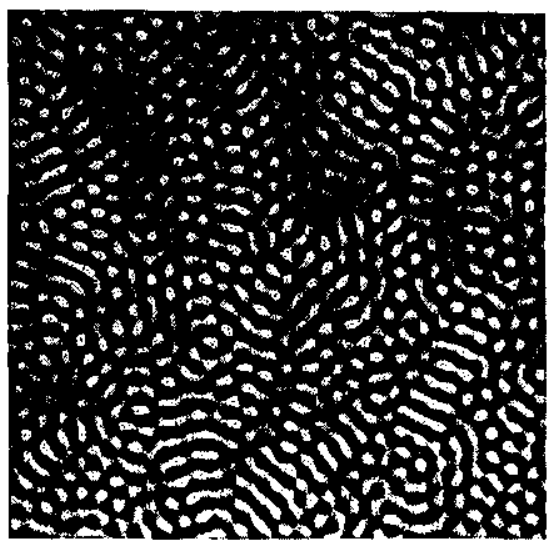

(c)

FIG. 6. This sequence of photographs illustrates the relaxation of the system from a perturbation-enforced striped state to a chaotic state. The pictures were taken (a) $0 \mathrm{~h}$, (b) $2 \mathrm{~h}$, and (c) $30 \mathrm{~h}$ after a spatial perturbation (stripes) was withdrawn. The control parameters were fixed: [I $\left.\mathrm{I}^{-}\right]_{0}=3.0$ mM, $\left[\mathrm{Na}_{2} \mathrm{SO}_{4}\right]_{0}=4.5 \mathrm{mM},\left[\mathrm{CH}_{2}(\mathrm{COOH})_{2}\right]_{0}^{B}=7.0 \mathrm{mM},\left[\mathrm{ClO}_{2}^{-}\right]_{0}^{A}=18 \mathrm{mM},\left[\mathrm{H}_{2} \mathrm{SO}_{4}\right]_{0}^{\wedge}=0.5 \mathrm{mM},\left[\mathrm{H}_{2} \mathrm{SO}_{4}\right]_{0}^{\mathrm{B}}=8.5 \mathrm{mM}$, temperature $=6.0^{\circ} \mathrm{C}$. The region of each image shown is $5.8 \times 5.8 \mathrm{~mm}^{2}$.

time scale much shorter than the time scale for the motion of grain boundaries in the stationary hexagonal or striped patterns. Both the pattern within a domain and the domain size continuously fluctuate. For example, a domain filled with dots may increase in size as it expands into neighboring striped regions, or decrease in size as its neighbors intrude.

The observed chemical turbulence is fundamentally different from spatiotemporal chaos observed previously for front structures; ${ }^{22,23}$ the latter are temporally chaotic but spatially highly coherent. In contrast, the turbulent patterns have no long range spatial order. Since the characteristic time for the evolution of the turbulent patterns is quite long, at least several hours, we have not yet analyzed the time dependence of these patterns, but it nonetheless seems clear that these patterns cannot be described by a low-dimensional strange attractor.

The observed chemical turbulence is not a transient phenomenon but an asymptotic stable state. This was proved by the following experimental procedure: Starting from a state of chemical turbulence, we perturbed the photo-sensitive system by illuminating it with intense light in a pattern of light and dark stripes. The wavelength of the perturbation was chosen to be the same as the intrinsic wavelength of the chemical patterns. After $30 \mathrm{~min}$ of perturbation, an almost regular pattern with few defects had formed, as shown in Fig. 6(a). As soon as the external perturbation was removed, defects began to grow. Figure 6(b) shows the pattern after $2 \mathrm{~h}$; defects have cut the system into small regions of dots and stripes. The turbulent state was fully recovered by $30 \mathrm{~h}$ after the perturbation was removed, as Fig. 6(c) illustrates.

The transition from a striped to a turbulent pattern was studied using the malonic acid concentration in compartment B $\left(\left[\mathrm{CH}_{2}(\mathrm{COOH})_{2}\right]_{0}^{\mathrm{B}}\right)$ as a control parameter. Below a critical value of the malonic acid concentration $(10.3 \mathrm{mM})$, the striped state becomes unstable and a tran- sition to a turbulent state occurs: Numerous defects emerge and cut the large domains of stripes into smaller ones, as the photographs in Fig. 7 illustrate. The image below each photograph shows the locations of the calculated grain boundaries of regions of dots and stripes, as will be explained later. After the transition, regions of dots appear everywhere in the system, as well as at the grain boundaries, and both the patterns within the domains and the domain boundaries start to evolve continuously.

Figure 8 presents measurements that characterize the transition: the total length of grain boundaries $(L)$, the average speed of pattern evolution $(v)$, the correlation length $(\xi)$, and the wavelength of the patterns $(\lambda)$ were each determined as a function of malonic acid concentration. For each measurement all control parameters were held fixed for a long enough time ( 24 to $50 \mathrm{~h}$ ) to make sure that the behavior was asymptotic. The wavelength of the striped pattern decreases as the transition point is approached, while the wavelength in the turbulent regime is almost independent of malonic acid concentration; see Figs. 7 and 8 . The wavelength was determined from the spatial Fourier transforms. We now describe how $L, \xi$, and $v$ were computed.

The boundaries of the domains were located using the following procedure: We first computed a spatial correlation between a given digital image and the function

$$
g(\mathbf{r})=\exp \left(i \mathbf{k}_{0} \cdot \mathbf{r}\right) \exp \left(-r^{2} / 2\right)
$$

where $\mathbf{k}_{0}$ is a vector whose magnitude was set equal to the intrinsic wave number of the patterns and whose direction was considered as a variable ( $r$ was measured in units of pixels; there were typically 20 pixels per wavelength). For a given $\mathbf{k}_{0}$, only patterns whose wave vector equals $\mathbf{k}_{0}$ would appear in the correlation function. We then low-pass filtered this correlation function, set a threshold, and obtained iso-value lines which represent the grain boundaries 
(a)
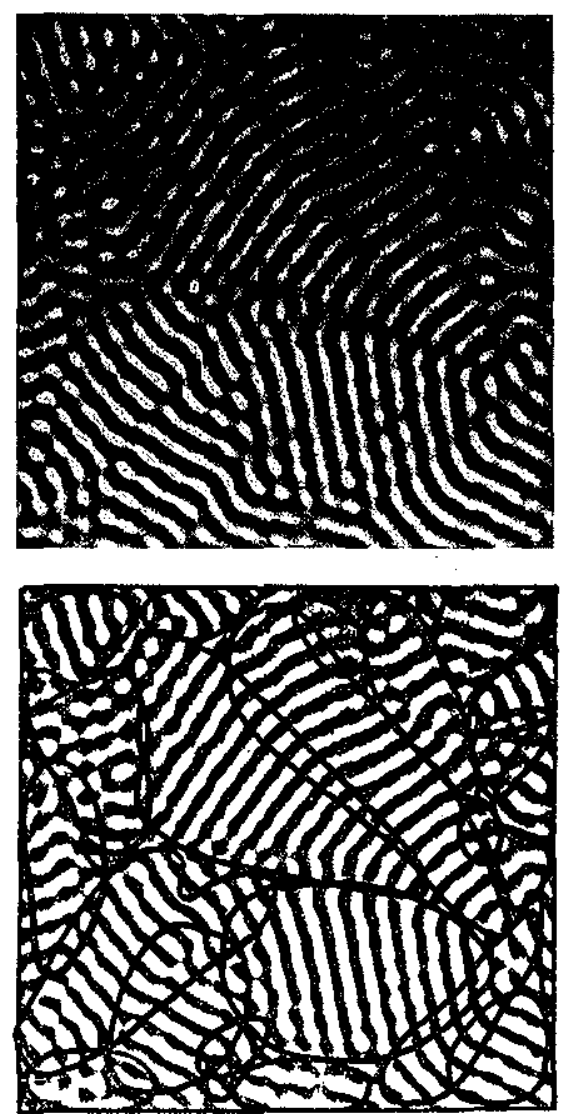

(d) (b)
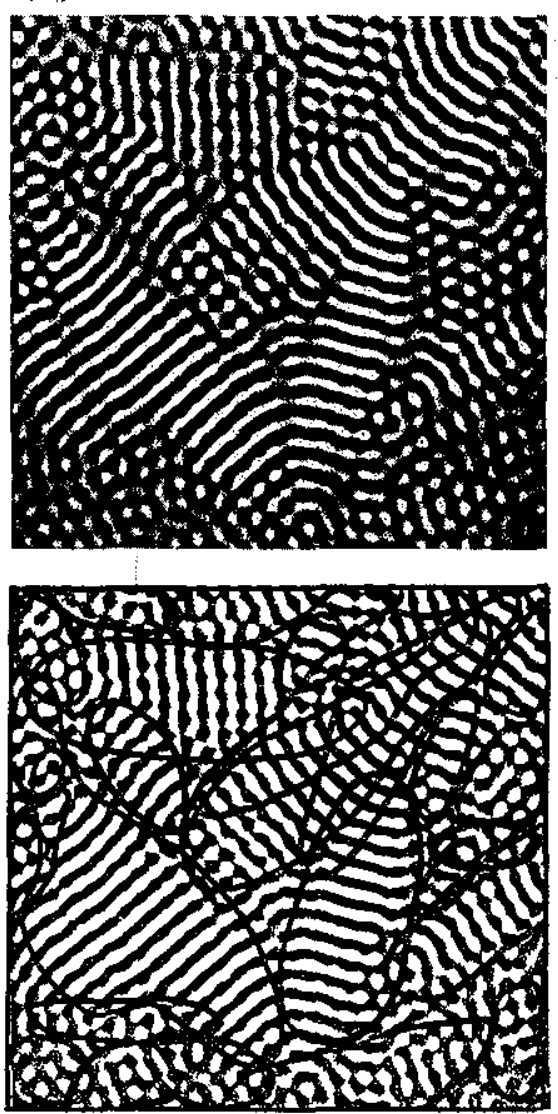

(e) (c)
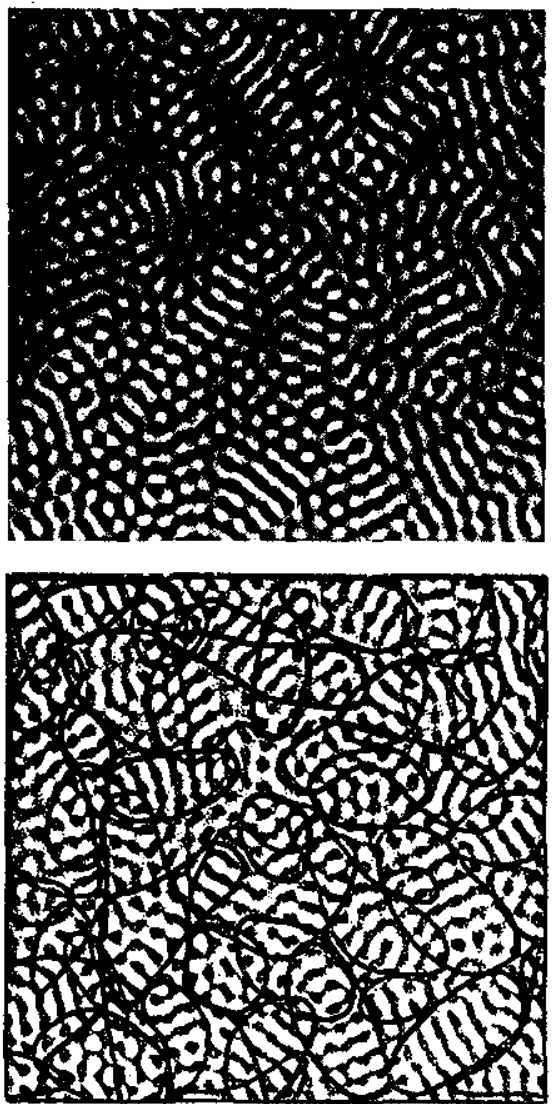

(f)

FIG. 7. Images (a)-(c) illustrate the transition from a striped pattern to chemical turbulence as the malonic acid concentration in compartment $B$ was decreased. Images (d)-(f) show the calculated grain boundaries of each image. The concentration of malonic acid in compartment $\mathrm{B}$ was (in $\mathrm{mM}$ ): (a) and (d), 12, stripes; (b) and (e), 10, stripes; (c) and (f), 8, turbulence. Other control parameters were held fixed: $\left[\mathrm{I}^{-}\right]_{0}=3.0 \mathrm{mM},\left[\mathrm{Na}_{2} \mathrm{SO}_{4}\right]_{0}=4.5 \mathrm{mM}$, $\left[\mathrm{ClO}_{2}^{-}\right]_{0}^{\mathrm{A}}=18 \mathrm{mM},\left[\mathrm{H}_{2} \mathrm{SO}_{4}\right]_{0}^{\mathrm{A}}=0.5 \mathrm{mM},\left[\mathrm{H}_{2} \mathrm{SO}_{4}\right]_{0}^{\mathrm{B}}=8.5 \mathrm{mM}$, temperature $=5.8^{\circ} \mathrm{C}$. The region shown is $5.8 \times 5.8 \mathrm{~mm}^{2}$.

of the patterns. Changing the direction of $\mathrm{k}_{0}$, we obtained grain boundaries of patterns with all orientations. Figure 7 (d)-(f) shows some grain boundaries calculated for the images of Fig. 7(a)-(c). Note that some domains overlap, which indicates that the calculated $L$ is proportional to rather than equal to the total length of the grain boundaries.

The correlation length $\xi$ of a state was calculated from the intensity autocorrelation function of a given digital image. The correlation function envelope of the measured intensity $I(\mathbf{R})$ was fit to an exponential,

$$
C(r)=\langle I(\mathbf{R}) I(\mathbf{R}+\mathbf{r})\rangle_{\mathbf{R}, \theta}=A e^{-r / \xi},
$$

where the vector $\mathbf{r}$ separating two points has magnitude $r$ and direction $\theta$; the averaging was done for all origins $\mathbf{R}$ in the pattern. Examples of correlation functions for a striped state and a turbulent state are presented in Fig. 9, and results for $\xi$ as a function of a control parameter are shown in Fig. 8. The correlation length for the stripes is longer than that for chemical turbulence, but in our experiments the correlation length was found to be less useful than $L$ and $v$ for distinguishing ordered and turbulent states.
The average speed of patterns $v$ was estimated by subtracting two images separated in time by a constant interval $(0.5 \mathrm{~h})$, calculating the root-mean-square deviation of the amplitudes, and averaging this deviation over all space. The result is directly proportional to the average speed of pattern motion if the time interval between two images is much less than the time for the system to move one wavelength, which was the case in our experiment.

The measurements in Fig. 8 show that the transition from a striped to a turbulent pattern transition occurred at $[\mathrm{MA}]_{0}^{\mathrm{B}}=10.3 \pm 0.3 \mathrm{mM}$ for both increasing and decreasing malonic acid concentration. Within the fairly coarse mesh $(0.5 \mathrm{mM}$ malonic acid) there was no hysteresis. Also, no significant critical slowing down was observed. The transition was marked by a rapid change in grain boundary length $L$ and average pattern speed $v$, while the correlation length $\xi$ and wavelength $\lambda$ changed gradually.

The phase diagram shown in Fig. 10 suggests that the turbulent state, which is located between hexagonal and striped regimes in the phase plane, can be considered as a time-dependent mixture of hexagons (dots) and stripes. As we have discussed, the transition from stripes to turbulence 


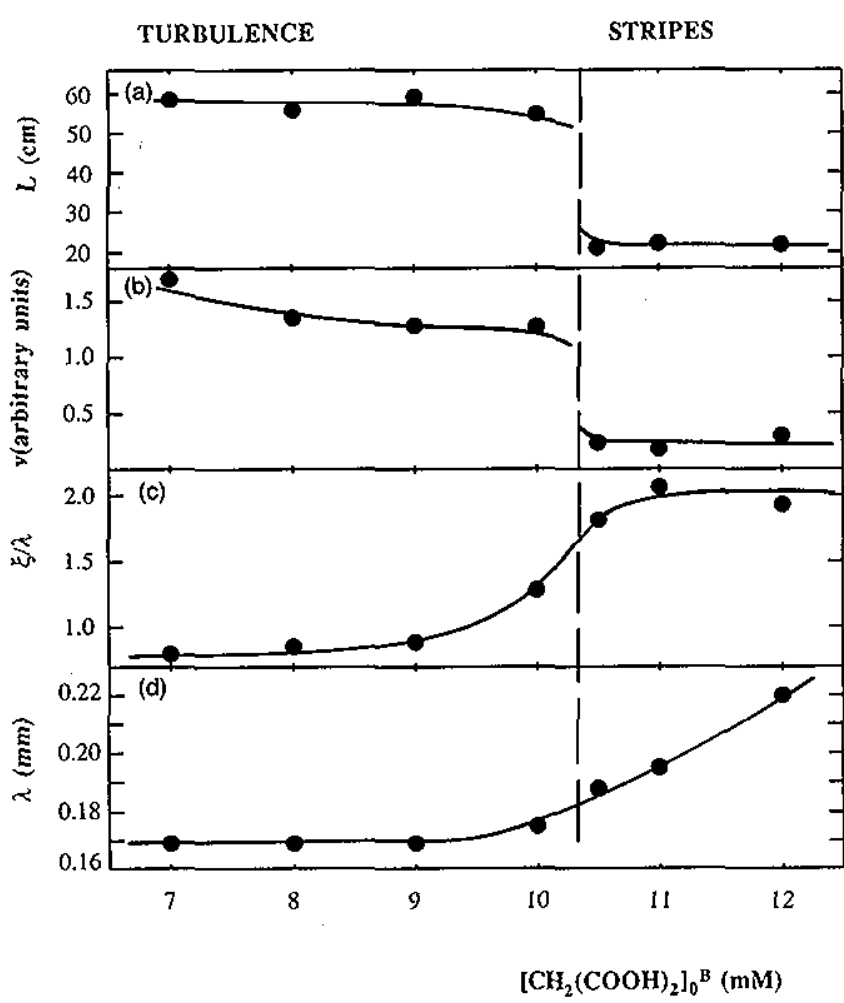

FIG. 8. The transition from stripes to chemical turbulence as a function of malonic acid concentration in compartment $B$ as characterized by the following quantities: $L$, the total length of the calculated grain boundaries; $v$, the average speed of pattern motion; $\xi$, correlation length; and $\lambda$, wavelength of the pattern. Other control parameters were held fixed at the values given for Fig. 7 .

is quite well defined. In contrast, the transition from hexagons to turbulence is ill-defined: $L, v, \xi$, and $\lambda$ all change gradually - the transition marked by the dashed line in Fig. 10 was arbitrarily defined as the point at which the average size of a domain of hexagons became smaller than $5 \lambda$.

\section{DISCUSSION}

Pattern formation in two-dimensional systems has been extensively studied using general equations that describe the amplitude of the patterns. ${ }^{37}$ In the absence of specific symmetries, which is the usual situation in chemistry, the primary symmetry breaking bifurcation leads to a hexagonal state. ${ }^{38-40}$ The secondary bifurcation may lead to a striped or a rhombic pattern, which for some range of control parameters coexists with hexagons (hysteresis) ${ }^{38-40}$ Our experimental results are consistent with the prediction that the first transition leads to hexagons, but we have not found a region of bistability of hexagons and stripes nor have we observed rhombic patterns; instead, under some conditions, we have observed a turbulent state in the parameter region between the hexagons and stripes.

The nature of the observed stationary mixed hexagonal-striped states is not clear at present. Buzano and Golubitsky ${ }^{41}$ found similar patterns in their analysis of amplitude equations. However, neither their calculations nor

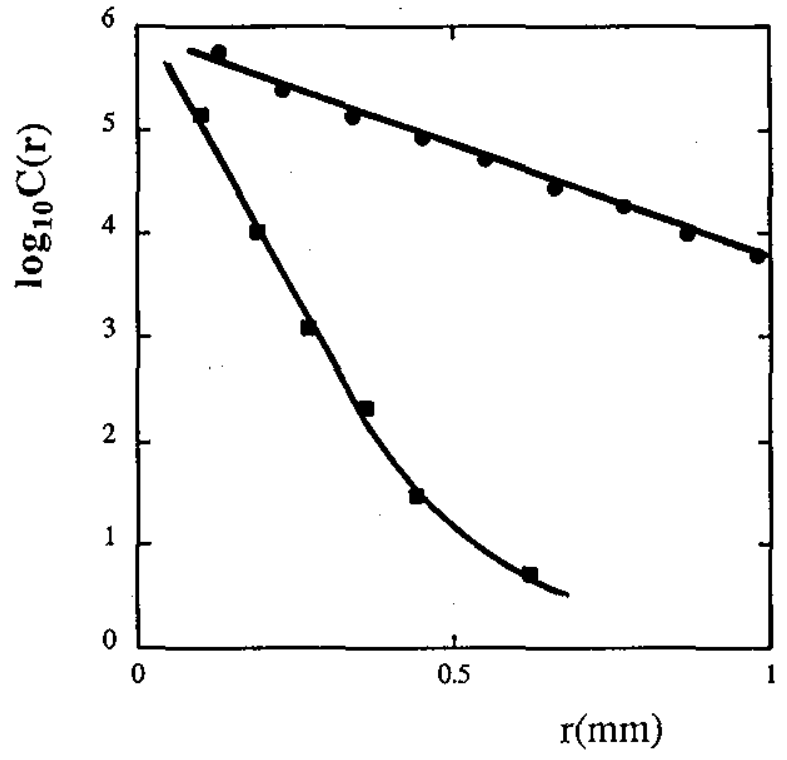

FIG. 9. A comparison of the envelopes of intensity autocorrelation functions for turbulence $(\boldsymbol{E})$ and stripes ( $)$. The resultant correlation lengths $\xi / \lambda$ of the turbulent and striped states are 0.85 and 2.1 , respectively. The concentration of malonic acid in compartment B for the turbulence and stripes was $9 \mathrm{mM}$ and $12 \mathrm{mM}$, respectively. Other control parameters were held fixed at the values given for Fig. 7 .

our experiments can prove that the mixed patterns are stable; they could be slowly decaying transient states that would eventually evolve into hexagons or stripes.

Theoretical studies of defect-mediated turbulence in two-dimensional systems have generally followed two approaches. One starts with local oscillators that couple through a diffusion process; ${ }^{10}$ another starts from a stationary patterned state. ${ }^{7}$ The first approach was taken by Kuramoto, ${ }^{10}$ who showed that if a system of oscillators is coupled by diffusion, a globally oscillating state can lose its

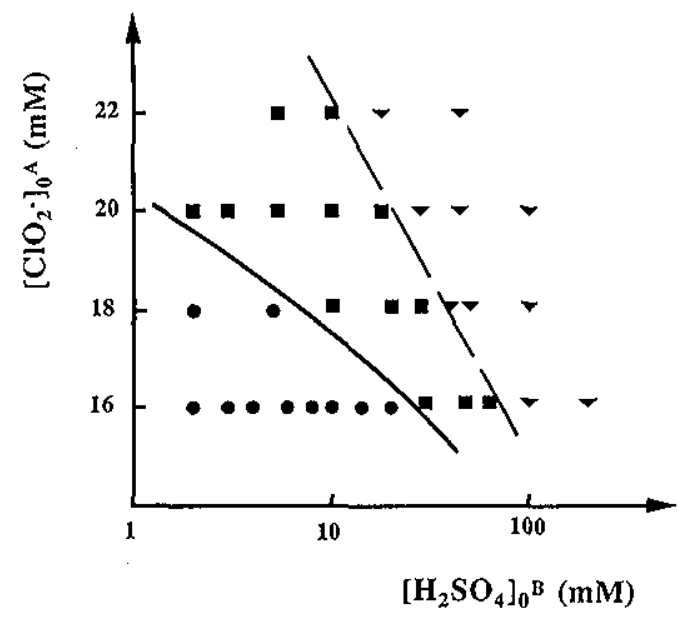

FIG. 10. Phase diagram showing stripes ( $)$, turbulence ( $\mathbf{\square}$ ), and hexagons $(\boldsymbol{\nabla})$ in the $\left(\left[\mathrm{ClO}_{2}^{-}\right]_{0}^{\mathrm{A}},\left[\mathrm{H}_{2} \mathrm{SO}_{4}\right]_{0}^{\mathrm{B}}\right)$ plane for the chlorite-iodidemalonic acid reaction in the disk gel reactor. The following control parameters were held fixed: $\left[\mathrm{I}^{-}\right]_{0}=3.0 \mathrm{mM},\left[\mathrm{Na}_{2} \mathrm{SO}_{4}\right]_{0}=4.5 \mathrm{mM}$, $\left[\mathrm{H}_{2} \mathrm{SO}_{4}\right]_{0}^{\mathrm{A}}=0.5 \mathrm{mM},\left[\mathrm{CH}_{2}(\mathrm{COOH})_{2}\right]_{0}^{\mathrm{B}}=9 \mathrm{mM}$, temperature $=6.2^{\circ} \mathrm{C}$. 
stability and undergo a transition to a state of phase turbulence, which is described by a "phase turbulence equation." Coullet et al. ${ }^{6}$ showed that phase turbulence in a sufficiently extended system leads to the creation of topological defects; thus phase turbulence can be considered as defect-mediated turbulence.

The second approach was taken by Eckmann and Procaccia, ${ }^{7}$ who established the following generic scenario: "Upon crossing the secondary instability, the fundamental (stationary) cellular pattern destabilizes in favor of another, spatially biperiodic stationary solution. The biperiodic stationary solution can be shown to exist within perturbation theory, but in truth it is destroyed in favor of spatially chaotic solutions by a mechanism of the Kolmogorov-Arnol'd-Moser (KAM) type." The authors also showed that if the underlying equations possess continuous symmetries that give rise to a secondary longwavelength instability, then randomly distributed defects will develop in the system.

Our data do not distinguish in an obvious way between these two approaches. We do not observe any hint of global oscillations in the neighborhood of the transition from hexagons to turbulence, as would be expected in the first scenario. Nor do we observe any indication of a spatially biperiodic pattern, as would be expected in the second scenario.

Another possibility is suggested by the observation that the turbulent state lies between hexagonal and striped regimes in the phase diagram (Fig. 10). In an analysis of amplitude equations, Ciliberto et al. ${ }^{42}$ showed that a grain boundary between two oblique rolls (stripes) gives rise locally to hexagons, and that, in the defect of hexagons, a roll (stripe) is present. We conjecture that in a parameter region where stripes and hexagons are nearly equally stable, competition between these orientationaliy degenerate patterns can lead to a state that wanders between hexagons and stripes and never settles down. Additional mathematical tools are needed to distinguish among the different possible mechanisms leading to defect-mediated turbulence.

In summary, we have presented experimental evidence of hexagonal, striped, and mixed Turing-type stationary patterns in a reaction-diffusion system that can be maintained indefinitely in a well-defined nonequilibrium state. We have also found a transition from the stationary patterns to disordered patterns that continuously evolve; this is the first experimental evidence of defect-mediated turbulence in a reaction-diffusion system.

In the discussion, we have considered the patterns to be two-dimensional, but a complete analysis must consider possible three-dimensional effects ${ }^{43}$ since the thickness of our gel is 6-12 times larger than the wavelength of the pattern. Moreover, the presence of the imposed concentration gradient normal to the plane of the pattern could possibly lead to a bifurcation route to turbulence that is qualitatively different from that of two-dimensional systems. Clearly more theoretical and experimental work is needed to understand how spatiotemporal patterns form and evolve in reaction-diffusion systems.

\section{ACKNOWLEDGMENTS}

We acknowledge fruitful discussions with A. Arneodo, J. Boissonade, P. Borckmans, P. De Kepper, G. Gunaratne, J. Lega, W. D. McCormick, Z. Noszticzius, D. Walgraef, and A. T. Winfree. This work was supported by the U.S. Department of Energy Office of Basic Energy Sciences and BP Venture Research.

${ }^{1}$ V. Steinberg, E. Moses, and J. Fineberg, Nucl. Phys. B (Proc. Suppl.) 2, 109 (1987).

${ }^{2}$ A. Pocheau, V. Croquette, and P. Le Gal, Phys. Rev. Lett. 55, 1094 (1985).

${ }^{3}$ R. Ribotta and A. Joets, in Cellular Structures and Instabilities, edited by J. E. Wesfried and S. Zaleski (Springer-Verlag, Berlin, 1984).

${ }^{4}$ N. B. Tufillaro, R. Ramshankar, and J. P. Gollub, Phys. Rev. Lett. 62, 422 (1989).

5. P. Gollub and R. Ramshankar, in New Perspectives in Turbulence, edited by S. Orszag and L. Sirovich (Springer-Verlag, Berlin, 1990).

${ }^{6} \mathrm{P}$. Coullet, L. Gil, and J. Lega, Phys. Rev. Lett. 62, 1619 (1989).

${ }^{7}$ J.-P. Eckmann and I. Procaccia, Phys. Rev. Lett. 66, 891 (1991).

${ }^{8} \mathrm{G}$. Nicolis and I. Prigogine, Self-organization in Nonequilibrium Chemical Systems (Wiley, New York, 1977).

${ }^{9}$ H. Haken, Synergetics, an Introduction (Springer-Verlag, Berlin, 1977).

${ }^{10} \mathrm{Y}$. Kuramoto, Chemical Oscillations, Waves, and Turbulence (SpringerVerlag, Berlin, 1984).

${ }^{1} \mathrm{R}$. J. Field and M. Burger (Eds.), Oscillations and Traveling Waves in Chemical Systems (Wiley, New York, 1985).

${ }^{12}$ A. T. Winfree, The Geometry of Biological Time (Springer, Berlin, 1980); When Time Breaks Down (Princeton U.P., Princeton, NJ, 1987).

${ }^{13} \mathrm{H}$. Meinhardt, Models of Biological Pattern Formation (Academic, New York, 1986).

${ }^{14}$ A. Babloyantz, Molecules, Dynamics and Life (Wiley, New York, 1986).

${ }^{15}$ J. D. Murray, Mathematical Biology (Springer-Verlag, New York, 1989).

${ }^{16}$ Interesting irregular chemical patterns have been observed recently in experiments on the Belousov-Zhabotinsky reaction in an inhomogeneous medium in a closed system: J. Maselko and K. Showalter, Physica D 49, 21 (1991).

${ }^{17}$ A. M. Turing, Philos. Trans. R. Soc. London Ser. B 327, 37 (1952).

${ }^{18}$ V. Castets, E. Dulos, J. Boissonade, and P. De Kepper, Phys. Rev. Lett. 64, 2953 (1990).

${ }^{19} \mathrm{~J}$. Boissonade, V. Castets, E. Dulos, and P. De Kepper, in Bifurcation and Chaos: Analysis, Algorithms, Applications, edited by T. Küpper, F. W. Schneider, R. Seydel, and $\mathrm{H}$. Troyer, International Series of $\mathrm{Nu}-$ merical Mathematics (Birkhauser, Basel, 1991), Vol. 97, p. 67.

${ }^{20} \mathrm{P}$. De Kepper, V. Castets, E. Dulos, and J. Boissonade, Physica D 49, 161 (1991).

${ }^{21}$ Q. Ouyang and H. L. Swinney, Nature 352, 610 (1991); see also A. T. Winfree, Nature 352, 568 (1991).

${ }^{22}$ W. Y. Tam, J. A. Vastano, H. L. Swinney, and W. Horsthemke, Phys. Rev. Lett. 61, 2163 (1988).

${ }^{23}$ W. Y. Tam and H. L. Swinney, Physica D 46, 10 (1990).

${ }^{24}$ A. Arneodo, J. Elezgaray, J. Pearson, and T. Russo, Physica D 49, 141 (1991).

${ }^{25}$ G. Kshirsagar, Z. Noszticzius, W. D. McCormick, and H. L. Swinney, Physica D 49, 5 (1991).

${ }^{26}$ P. De Kepper, J. Boissonade, and I. R. Epstein, J. Phys. Chem. 94, 6525 (1990).

${ }^{27}$ Q. Ouyang, V. Castets, J. Boissonade, J. C. Roux, P. De Kepper, and H. L. Swinney, J. Chem. Phys. 95, 351 (1991).

${ }^{28}$ M. Boukalouch, J. Boissonade, and P. De Kepper, J. Chim. Phys. (France) 84, 1353 (1988).

${ }^{29}$ Q. Ouyang, J. Boissonade, J. C. Roux, and P. De Kepper, Phys. Lett. A 134, 284 (1989).

${ }^{30} \mathrm{P}$. De Kepper, Q. Ouyang, J. Boissonade, and J. C. Roux, in Dynamics of Exotic Phenomena in Chemistry, edited by H. Beck and B. Körös, React. Kinet. Catal. Lett. (Budapest) 42, 275 (1990).

${ }^{31} \mathrm{~J}$. Boissonade, Q. Ouyang, A. Arneodo, J. Elezgaray, J. C. Roux, and P. De Kepper, in Nonlinear Wave Processes in Excitable Media, edited by 
A. V. Holden, M. Markus, and H. G. Othmer (Pergamon, New York, 1991), p. 47.

${ }^{32}$ S. C. Müller, T. Plesser, and B. Hess, Science 230, 661 (1985); J. Stat. Phys. 48, 991 (1987).

${ }^{33}$ J. J. Tyson and J. P. Keener, Physica D 32, 327 (1988).

${ }^{34}$ The two-dimensional spatial Fourier transforms were calculated for defect-free hexagonal, striped, ō mixed state regions such as those shown in Fig. 3(b)-(d). These defect-free patterns were localized in relatively small regions, typically 6 to 8 wavelengths of the patterns, while on a larger length scale the patterns usually had point defects and grain boundaries; the Fourier transforms of these patterns consisted of a circular ring around the origin instead of individual peaks.

${ }^{35}$ D. B. White, J. Fluid. Mech. 191, 247 (1988)

${ }^{36}$ R. C. DiPrima and H. L. Swinney, in Hydrodynamic Instabilities and the Transition to Turbulence, edited by H. L. Swinney and J. P. Gollub (Springer-Verlag, Berlin, 1981), p. 139.

${ }^{37}$ P. Manneville, Dissipative Structures and Weak Turbulence (Academic, Boston, 1990).

${ }^{38}$ L. M. Pismen, J. Chem. Phys. 72, 1900 (1980).

${ }^{39}$ F. H. Busse, J. Fluid. Mech. 30, 625 (1967).

${ }^{40}$ B. A. Malomed and M. I. Tribel'skii, Sov. Phys. JETP 65, 305 (1987).

${ }^{41}$ E. Buzano and M. Golubitsky, Phil. Trans. R. Soc. London Ser. A 308, 617 (1983).

${ }^{42}$ S. Ciliberto, P. Coullet, J. Lega, E. Pampaloni, and C. Perez-Garcia, Phys. Rev. Lett. 65, 2370 (1990).

${ }^{43}$ A. DeWit, G. Dewel, P. Borckmans, and D. Walgraef, Physica D (to appear). 